\title{
BESPRECHUNGSAUFSÄTZE
}

\section{Regimewechsel und Demokratisierung in Lateinamerika}

\author{
Von Nikolaus Werz
}

Emilio García Méndez, Recht und Autoritarismus in Lateinamerika. Argentinien, Uruguay und Chile 1970-1980, Frankfurt a.M., Vervuert, 1985, 280 S., DM 29,80

Guillermo O'Donnell, Philippe C. Schmitter, Laurence Whitehead (Hrsg.), Transitions from Authoritarian Rule. Comparative Perspectives, London, The John Hopkins University Press, 1986, 190 S., \$ 12.05

Viktor Sukup, Zeitbombe Südamerika. Ein Kontinent zwischen Diktatur und Demokratie, Köln, Pahl-Rugenstein, 1988, 269 S., DM 14,80

James M. Malloy and Mitchell A. Seligson (Hrsg.), Authoritarians and Democrats. Regime Transition in Latin America, Pittsburgh 1987, University of Pittsburgh Press, 268 S., $\$ 12.95$

Die Re-Demokratisierung politischer Regierungsformen in den südamerikanischen Staaten liegt bereits einige Jahre zurück. Mittlerweile sind eine Reihe von Studien zu dem Thema erschienen, die sich zunächst mit den Regimewechseln und später mit dem Problem der Konsolidierung der Demokratie auseinandersetzten. Die ersten Arbeiten zu diesen Fragen entstanden in den USA. In zunehmendem Maße sind nun auch Publikationen zu dem Gebiet in der BRD veröffentlicht worden.

In dem ersten der hier zu besprechenden Bücher geht es um den Autoritarismus der 70er Jahre. Der Autor konstatiert in der staatstheoretischen Literatur über Lateinamerika eine Forschungslücke zu den rechtlichen Fragen. Ziel der Studie von García Méndez ist es, "die Besonderheit und die Funktionen der Rechtsinstanz im Rahmen der politisch-rechtlichen Struktur der sich in einer Krise befindenden Herrschaftssysteme zu verdeutlichen und zu analysieren" (S. 13). Hauptthemen der Arbeit seien, heißt es dann an anderer Stelle, "der Autoritarismus und seine Institutionalisierung" (S. 26). 
Die Untersuchung gliedert sich in acht Kapitel, in denen es nicht allein um die rechtliche Thematik geht, sondem eine Reihe weiterer politischer und theoretischer Aspekte behandelt werden. Für den Leser wird die Beziehung zwischen den Abschnitten über weite Strecken nicht deutlich. So geht es in den Kapiteln I-V um den Begriff des Autoritarismus, den Zusammenhang von Kapitalismus und Autoritarismus, das Verhältnis von Zentrum zu Peripherie und die Doktrin der inneren Sicherheit. Die gemeinhin als "Doktrin der nationalen Sicherheit" bezeichnete Ideologie der neueren Militärregime wird hier "Doktrin der inneren Sicherheit" (S. 83 ff.) genannt, ohne daß diese Veränderung genauer erklärt würde. In Kapitel V werden unvermittelt "einige Aspkete der Theorie Niklas Luhmanns" mit der sogenannten Doktrin der inneren Sicherheit in Verbindung gesetzt und kritisiert. Erst im zweiten Teil der Arbeit, d.h. ab Kapitel VI, wird die autoritäre Institutionalisierung im 'cono sur' Gegenstand der Untersuchung. Im Falle Chiles zeige das Fehlen "einer wirklichen Institutionalisierung den autoritären und nicht faschistischen oder totalitären Charakter des Regimes" (S. 186). Kapitel VII gibt einen Úberblick der Arbeiten zu Staatstheorie und Rechtsinstanz in Lateinamerika, und das Schlußkapitel behandelt dann das Verhältnis von politischer Herrschaft und juristischer Hegemonie. Es fehlt eine Zusammenfassung der Ergebnisse am Ende des Buches, das bereits zum Zeitpunkt seiner Publikation durch die neuere politische Entwicklung überholt war. Ebensowenig ist es geeignet, einen Überblick zu "Recht und Autoritarismus" während der 70er Jahre in ausgewählten südamerikanischen Staaten zu vermitteln.

Das von Guillermo O' Donnell und anderen herausgegebene Buch ist Teil einer Serie, die in vergleichender Perspektive den Úbergang vom Autoritarismus zur Demokratie in Südeuropa und Lateinamerika untersucht. Einige der Herausgeber - wie O'Donnell und Schmitter - hatten sich in den 70er Jahren mit dem Zusammenbruch demokratischer Regime, der sogenannten Break-Down-Forschung, auseinandergesetzt. Im Zuge der politischen Veränderungen in Südeuropa und Südamerika haben sie sich nun der Re-Demokratisierung zugewandt.

Die in der Studie vereinigten Aufsätze gehen auf eine Vortragsreihe am Woodrow Wilson Center in Washington aus den Jahren 1979-1981 zurück. In seinem Beitrag "International Aspects of Democratization" analysiert Laurence Whitehead die Politik der USA und der europäischen Staaten zur Förderung der Demokratie im internationalen Maßstab. Er konstatiert, daß die europäischen Demokratie-Definitionen das Schwergewicht mehr auf soziale und ökonomische Partizipation legen, während für die Nordamerikaner das Kriterium freier Wahlen im Vordergrund steht. Obwohl der Autor der nordamerikanischen Außenpolitik in Lateinamerika viel Verständnis entgegenbringt, muß er feststellen, "daß es einfacher ist, Beispiele zu finden, in denen der US-Einfluß zur Schwächung gewählter Regierungen beigetragen hat, als umgekehrt" (S. 38). Im Vergleich schneiden die europäischen Staaten bei der Demokratieförderung wesentlich besser ab, zumal es in den 70er Jahren gelang, die parlamentarische Demokratie in ganz Europa zu festigen. Whitehead betont die Rolle der Sozialistischen Internationale und ihrer Mitgliederparteien in Europa bei der 
Unterstützung des Kampfes gegen die Diktaturen in Spanien und Griechenland. Ein Grund für die Akzeptanz liberaler Demokratie durch die Oberschichten in Griechenland und den iberischen Gesellschaften war die Aussicht, mit dem Eintritt in die Europäische Gemeinschaft auch an deren Wohlstand teilhaben zu können. Solche Bedingungen sind in Lateinamerika nicht gegeben. Die Ausdehnung der europäischen Aktivitäten und der von dort operierenden internationalen Parteienverbände auf Lateinamerika werden detailliert und zuweilen etwas langatmig beschrieben, die Aussichten auf einen Erfolg dort jedoch wesentlich zurückhaltender beurteilt.

Adam Przeworski setzt sich mit theoretischen Problemen beim Ubergang zur Demokratie auseinander. Er unterscheidet einerseits zwischen makro-orientierten Studien, die die Demokratie als Frucht eines bestimmten ökonomischen Entwicklungsstandes betrachten, wobei er die klassische Studie von Barrington Moore als Prototyp bezeichnet; davon hebt er andererseits mikro-orientierte Arbeiten ab, die das Verhalten politischer Regime in konkreten historischen Situationen untersuchen.

In einem vergleichenden Artikel skizziert Alfred Stepen in acht Schritten das Ende autoritärer Regime. Die drei ersten gelten für Staaten, in denen ein verlorener Krieg Anlaß für den Regimewechsel war, womit die europäischen Länder und Japan nach dem Zweiten Weltkrieg gemeint sind. In den lateinamerikanischen Gesellschaften sind mehr die innenpolitischen Faktoren und in gewisser Hinsicht auch außenpolitischer Druck für das Gelingen von Regimewechseln hin zu demokratisch gewählten Regierungen verantwortlich. Robert $R$. Kaufmann behandelt den Prozeß der Liberalisierung und Demokratisierung in ausgewählten südamerikanischen Staaten. Er untersucht die politischen Koalitionen, die sich im Zuge der Auflösung autoritärer Regime ergeben, und spekuliert über mögliche politische Optionen und Alternativen in Südamerika.

"Es ist leichter, die Regierung zu demilitarisieren als die Zentren der Macht", konstatiert Alain Rouquié in seinem Aufsatz über "Demilitarization and the Institutionalization of Military-Dominated Politics in Latin America". Der französische Wissenschaftler schätzt die Chancen einer effektiven Kontrolle des Militärs durch die Zivilisten nüchtern und skeptisch ein. Zwei Aufsätze zu ökonomischen Fragen - Fernando-H. Cardoso über die Rolle der brasilianischen Unternehmer im Prozeß des Übergangs, John Sheahan über die wirtschaftlichen Rahmenbedingungen der Re-Demokratisierung - stehen am Ende des Buches, das mit Ausnahme der drei mehr theoretisch gehaltenen Anfangsbeiträge durch die jüngere Entwicklung in Südamerika etwas überholt erscheint. Wenn indessen der Zeitpunkt der Entstehung der Beiträge berücksichtigt wird (Anfang der 80er Jahre), dann belegt der Band trotz der relativ späten Veröffentlichung einmal mehr, daß sich Sozialwissenschaftler in den USA schneller mit diesen Themen auseinandergesetzt haben als die meisten deutschen Lateinamerikaforscher.

Im Unterschied zu den anderen hier besprochenen Studien oder Aufsatzsammlungen versucht das Buch von Viktor Sukup eine Gesamtdarstellung zu geben. Sowohl der Titel "Zeitbombe Südamerika. Ein Kontinent zwischen Diktatur und Demokratie" als auch das viel- 
deutige Titelphoto (ein Frauengesicht hinter dunkler Sonnenbrille) deuten darauf hin, da $\beta$ ein größeres Publikum angesprochen werden soll. Dem Verfasser, ein österreichischer Soziologe, der mehrere Jahre in Südamerika gelebt und an Universitäten unterrichtet hat, geht es weniger um die politischen Aspekte als um die sozio-ökonomischen Zusammenhänge (S. 7). Das Buch untersucht zwischen einem Eingangs- und einem Schlußkapitel die Lage in "zehn Ländem zwischen Diktatur und Demokratie". Im ersten Kapitel, "Demokratisierung trotz Wirtschaftskrise - eine paradoxe Logik in historischstruktureller Perspektive", gibt Sukup einen Abriß der historischen Entwicklung Südamerikas. Dabei schwankt die Darstellung zwischen einer traditionellen Dependencia-Perspektive, so in dem nur sechs Seiten umfassenden Abschnitt "Südamerika in der internationalen Arbeitsteilung: Von Kolumbus bis Roosevelt", und dem gängigen Hinweis auf die Pluralität der Interpretationen, "weil es nicht eine 'Erklärung' für äußerst komplizierte sozio-ökonomische und historische Prozesse gibt" (S. 27). An anderer Stelle der Einleitung konstatiert er, daß durch die Krise "das Entwicklungsmodell im allgemeinen mehr in Frage gestellt ist als die 'Diktatur' oder 'Demokratie' an sich" (S. 49), ein Aspekt, der - wie der Titel - im Schlußteil nicht wieder aufgegriffen wird.

Das schwierige Vorhaben, die ökonomisch-politische Entwicklung Gesamt-Südamerikas zu charakterisieren, wird im Eingangs- und Schlußteil nicht eingelöst. Dazu wäre eine genauere sozialgeschichtliche Interpretation der gesellschaftlichen Veränderungen in den letzten beiden Jahrzehnten erforderlich gewesen, eine Aufgabe, die allerdings in der Lateinamerikaforschung noch aussteht. Wer indessen im Hauptteil des Buches liest ("Zehn Länder zwischen Diktatur und Demokratie"), kann zu den einzelnen Staaten auf knappem Raum einen Uberblick der neueren Geschichte und Politik erhalten. Welche Altemativen diskutiert der Autor, um die "Zeitbombe Südamerika" zu "entschärfen"? Sukup geht nicht direkt auf diese Frage ein, sondem behandelt abschließend lediglich die Integrations- und Kooperationsbestrebungen in Südamerika. In dem realistischeren Ansatz der Annäherung von Nachbarstaaten, wie ihn Argentinien, Brasilien und Uruguay in den letzten Jahren verfolgt haben, sieht er einen Fortschritt gegenüber dem früheren, utopischen "gemeinsamen Markt".

Um die in der ersten Hälfte der 80er Jahre stattgefundenen Regimewechsel und die Chancen der Demokratien geht es in dem Band von Malloy und Seligson. In seinem Einleitungsbeitrag weist Seligson darauf hin, daß demokratische Perioden in den 1820er und 1920er Jahren von kurzer Dauer waren. Die Aussichten der gegenwärtigen Demokratisierung schätzt er optimistischer ein: In neun von den zehn in dem vorliegenden Band behandelten Ländem seien die elementaren sozio-ökonomischen Voraussetzungen für eine stabile demokratische Entwicklung mittlerweile gegeben. Lediglich Bolivien bilde eine Ausnahme. Hinzu kämen weitere Gründe, die darauf hindeuteten, daß die aktuelle demokratische Phase von längerer Dauer sein werde als die vorherigen. Die Erfahrungen mit den jüngsten Militärdiktaturen seien so negativ gewesen, daß 1 . in Zukunft die Generäle nicht mehr mit der Unterstützung ziviler Partner rechnen könnten; 2. trotz der Professionalisierung die Militärs 
in Wirtschaftsfragen ähnlich erfolglos geblieben seien wie die von ihnen gestürzten Regierungen; 3. die zivilen Präsidenten zu einem Zeitpunkt an die Macht gelangten, zu dem die ökonomischen Voraussetzungen für die Demokratie deutlich günstiger seien als in der Vergangenheit. Neuere Untersuchungen zur politischen Kultur ausgewählter lateinamerikanischer Gesellschaften hätten darüber hinaus gezeigt, daß in Argentinien, Chile und Mexiko pro-demokratische Werte in der Bevölkerung weit verbreitet seien und die Wurzeln des Autoritarismus daher woanders gesucht werden müßten (S. 10).

Die Autoren der Länderbeiträge weichen nicht nur von Seligsons überraschend optimistischer Einschätzung der wirtschaftlichen Situation Lateinamerikas ab. So stellt Aldo C. Vacs fest, daß es in der argentinischen Gesellschaft nie einen politischen Pluralismus gegeben habe und daher erst die politische Kultur des Landes modifiziert werden müsse, um den Bestand der Demokratie zu sichem (S. 39). Duncan Baretta und Markoff sprechen in Anlehnung an einen Begriff von Juan Linz von einer "demokratischen Situation" und einer "semi-autoritären Demokratie" in Brasilien (S. 62-63). Angesichts der starken wirtschaftlichen und sozialen Probleme könne sich der Prozeß des Uberganges (spanisch: transición) in einen Dauerzustand verwandeln. Der Aufsatz über Chile gelangt zu dem Ergebnis, daß dort unter Pinochet ein spezifisches System mit neopatrimonialen Zügen entstanden sei, weswegen die Entwicklung anders verlaufen werde als in den Nachbarländern. Und in Bolivien habe die Demokratie bislang überlebt, weil die Militärs geschwächt seien und die politischen Führer unter demokratischen Herrschaftsformen weniger zu verlieren hätten (S. 118). Neben weiteren Länderartikeln (Peru, Ekuador) enthält das Buch zwei Aufsätze zur Entwicklung in Mittelamerika (Seligson, Rosenberg) sowie zum Thema "Die USA und die Demokratie in Lateinamerika". Abschließend faßt James Malloy in einem lesenswerten Aufsatz Merkmale einer "Politik des Uberganges in Lateinamerika" zusammen. Einen einheitlichen Trend zu Demokratie oder Diktatur gebe es nicht, besser lasse sich seit den 1930er Jahren von einem zyklischen Wechsel zwischen autoritären und demokratischen Herrschaftsformen sprechen. Etwa alle zwanzig Jahre werde der Trend zu Autoritarismus und Diktatur wieder durch den zur Demokratie abgelöst. Gemeinsam sei den Regimen die Tatsache gewesen, daß sie die ökonomischen Schwierigkeiten nicht lösen konnten, so daß die Regimewechsel aufgrund der Fehler des vorherigen Regimes stattfanden. Das lateinamerikanische politische System weise einen Kooptationscharakter auf und beruhe auf informellen Allianzen zwischen verschiedenen Machtgruppen unterhalb der demokratischen Oberfläche. Malloy verweist in diesem Zusammenhang auf die klassische Studie von Charles W. Anderson, "Politics and Economic Change in Latin America", 1967. Malloy bleibt nicht bei der Analyse der Regimeformen stehen, sondem sieht in der patrimonialen Sozialstruktur den Kem institutioneller Instabilität in Lateinamerika (S. 252). Entscheidend sei der Zugang zu den Machtquoten und der Verteilungskampf. Seit 1960 hätten sowohl die autoritären als auch die demokratischen Regime in einer Konfrontation mit den Gewerkschaften gestanden. Dieser Konflikt komme in dem Gegensatz Populismus und Antipopulismus zum Ausdruck. Die Ablösung der populistischen Regime durch antipopulistische 
Diktaturen und die emeute Hinwendung zu gewählten Präsidenten zeige, daß von einem regelrechten Zyklus gesprochen werden könne.

Die zeitgenössischen Ereignisse in Südamerika scheinen diese Interpretation, die von verschiedenen Politikwissenschaftlicher geteilt wird, zu bestätigen. Nach der Krise der Diktaturen gelangten in fast allen südamerikanischen Staaten liberal-demokratische Präsidenten an die Regierung. Sie betonten die Notwendigkeit parlamentarisch-präsidentieller Demokratie, unterschieden sich in der Wirtschaftspolitik allerdings kaum von dem neoliberalen Kurs der Militärs. Die hohen Erwartungen der Bevölkerung und der Intellektuellen in die "neuen Demokratien" begannen bald einer Emüchterung zu weichen. Die strafrechtliche Verfolgung der Menschenrechtsverletzungen wurde, sofern sie überhaupt begonnen hatte, unter dem Druck der Generäle eingestellt, die Verschuldung sowie der Schuldendienst stiegen weiter an, und von einigen Präsidenten begannen Fälle von Nepotismus und Mißwirtschaft bekannt zu werden, die schon in den 60er Jahren zur Diskreditierung der Demokratie in Lateinamerika beigetragen hatten. Gegenüber dem universalistischen Diskurs der amtierenden Präsidenten, von denen sich besonders Rául Alfonsín in Argentinen stark an Europa orientierte, erleben populistisch-nationalistische Politiker eine Renaissance. In Venezuela, Argentinien und Brasilien haben sie gute Chancen, die kommenden Wahlen zu gewinnen. Peru kann mit der Ablösung des konservativ-liberalen Belaunde Terry durch den zunächst populären Apristen Alan Garcia als Vorreiter gelten.

\title{
Demokratie und lateinamerikanische Gesellschaftsstrukturen
}

\author{
Von Wolfgang Spoerr
}

Manfred Mols, Demokratie in Lateinamerika, Stuttgart, Berlin, Köln, Mainz, Urban Taschenbücher, Kohlhammer, 1985, 220 S., DM 28,-

Wolfgang Dietrich, Honduras - ein Demokratisierungsversuch zwischen Repression, Revolution und Resignation, Reihe Heidelberger Dritte Welt Studien, Bd. 22, Heidelberger Verlagsanstalt und Druckerei, 1987, 321 S., DM 29,-

Ausgehend von der normativen Vorgabe des Konzeptes einer "offenen, pluralistischen Demokratie" westlichen Musters analysiert der Mainzer Politikwissenschaftler Manfred Mols deren Verwirklichungs- und Stabilisierungschancen. Konsequent für eine Studie, die 